\title{
MicroRNA-130a is upregulated in colorectal cancer and promotes cell growth and motility by directly targeting forkhead box F2
}

\author{
WENZHONG CHEN ${ }^{1,2}$, KEHUI TONG ${ }^{2}$ and JIREN YU ${ }^{1}$ \\ ${ }^{1}$ Department of Gastrointestinal Surgery, The First Affiliated Hospital of Zhejiang University, \\ Hangzhou, Zhejiang 310009; ${ }^{2}$ Department of Gastrointestinal Surgery, Yinzhou Hospital, \\ School of Medicine, Ningbo University, Ningbo, Zhejiang 315040, P.R. China
}

Received April 3, 2016; Accepted June 20, 2017

DOI: $10.3892 / \mathrm{mmr} .2017 .7257$

\begin{abstract}
Colorectal cancer (CRC) is one of the most prevalent cancers among males and females worldwide. Despite progress in diagnostic and therapeutic strategies for CRC patients, the prognosis for patients with advanced CRC remains poor. MicroRNAs (miRNAs/miRs) are a class of highly conserved short, endogenously expressed and single-stranded non-coding RNAs. In recent years, increasing studies have demonstrated that dysregulation of miRNAs is closely associated with CRC carcinogenesis and progression. The aim of the present study was to explore the expression, roles and underlying molecular mechanism of miR-130a in CRC. The results indicated that miR-130a was significantly upregulated in CRC, and that miR-130a expression levels were correlated with TNM stage and lymph node metastasis of CRC. Inhibition of miR-130a markedly suppressed colorectal cancer cell proliferation, migration and invasion. Furthermore, forkhead box F2 (FOXF2) was identified as a direct downstream target gene of miR-130a in colorectal cancer. Downregulation of FOXF2 could partially reverse the functions induced by miR-130a under-expression in CRC cells. These findings suggested that miR-130a can regulate FOXF2 and function as an oncogene in CRC. Therefore, miR-130a may serve as a useful therapeutic agent for miRNA-based CRC targeted therapy.
\end{abstract}

\section{Introduction}

Colorectal cancer (CRC) is one of the most prevalent cancers among males and females worldwide, with an estimated one million new cases and half a million mortalities each year $(1,2)$. Many risk factors contributing to CRC have been reported,

Correspondence to: Professor Jiren Yu, Department of Gastrointestinal Surgery, The First Affiliated Hospital of Zhejiang University, 79 Qingchun Road, Hangzhou, Zhejiang 310009, P.R. China

E-mail: zhejiang_jirenyu@163.com

Key words: microRNA-130a, colorectal cancer, growth, motility, forkhead box F2, microRNA-based targeted therapy including excessive alcohol use, obesity, age, genetic mutations and chronic intestinal inflammation (3). Currently, the usual therapeutic treatments for patients with CRC are surgery resection, followed by radiotherapy and chemotherapy (4). Despite progress in diagnostic and therapeutic strategies for $\mathrm{CRC}$, the prognosis for patients with advanced CRC remains poor (5). The 5-year overall survival rate for early CRC cases is $~ 90 \%$, but decreases to $<5 \%$ in advanced CRC patients with metastases or recurrence (6). Therefore, it is urgent to fully understand the mechanisms underlying carcinogenesis and progression of CRC, and investigate the novel therapeutic targets which may help to generate more effective therapies.

MicroRNAs (miRNAs/miRs) belong to a class of highly conserved short, endogenously expressed and single-stranded non-coding RNA molecules which are 22 nt long $(7,8)$. They negatively regulate gene expression through binding to specific complementary sites within the 3 ' untranslated regions (3'UTRs) of targeted mRNAs in a base-pairing manner, resulting in mRNA degradation or translational inhibition at the translational level (9-11). To date, $>1,400$ miRNAs have been identified, which accounts for 2-5\% of the whole human genome, and modulates the expression levels of $<20 \%$ of human genes (12). A variety of studies have reported that miRNAs have important roles in basic biological processes, including cell proliferation, apoptosis, differentiation, invasion, metastasis and angiogenesis (13-15). They may function as oncogenes or tumor suppressor genes in human cancers, depending on their target genes $(16,17)$. In recent years, increasing studies have demonstrated that dysregulation of miRNAs is closely associated with CRC occurrence and progression. For example, miR-542-3p inhibits the growth and invasion of CRC cells through targeted regulation of cortactin (18). Qiu et al (19) reported that miR-497 inhibits invasion and metastasis of CRC cells by targeting vascular endothelial growth factor-A. These findings emphasize the importance of miRNAs in CRC initiation and progression, and may serve as therapeutic targets for novel treatment strategies against CRC.

The present study identified miR-130a to be significantly upregulated in CRC tissues and cell lines. In addition, miR-130a expression levels were correlated with TNM stage and lymph node metastasis of CRC. Furthermore, miR-130a was demonstrated to be a novel negative regulator in the progression of CRC via directly targeting forkhead box F2 
(FOXF2). Therefore, the present study investigated the possibility that miR-130a may be an attractive candidate for miR-130a/FOXF2 based targeted therapy for patients with CRC.

\section{Materials and methods}

Human specimens. A total of 53 paired CRC and tumor adjacent tissues (TATs) were collected from the First Affiliated Hospital of Zhejiang University (Hangzhou, China) between 2011 and 2014, were used in the present study. These specimens were obtained from patients with CRC who underwent surgical resection without prior radiotherapy and chemotherapy, and immediately snap-frozen in liquid nitrogen and stored at $-80^{\circ} \mathrm{C}$. This study was approved by the Ethical Committee of the First Affiliated Hospital of Zhejiang University. Informed written consent was obtained from all patients.

Cell culture and transfection. The SW480, SW620, HCT116, HT29 and LoVo human CRC cell lines and the FHC human normal colon epithelium cell line were purchased from American Type Culture Collection (Manassas, VA, USA). All cell lines were maintained in Dulbecco's modified Eagle's medium (Gibco; Thermo Fisher Scientific, Inc., Waltham, MA, USA) supplemented with $10 \%$ fetal bovine serum (FBS, Gibco; Thermo Fisher Scientific, Inc.), $100 \mathrm{IU} / \mathrm{ml}$ penicillin (Gibco; Thermo Fisher Scientific, Inc.) and $100 \mu \mathrm{g} / \mathrm{ml}$ streptomycin (Gibco; Thermo Fisher Scientific, Inc.) at $37^{\circ} \mathrm{C}$ under $5 \% \mathrm{CO}_{2}$.

The miR-130a inhibitor, negative control (NC) inhibitor, FOXF2 small interfering (si)RNA and NC siRNA were synthesized and purified by Guangzhou RiboBio Co., Ltd. (Guangzhou, China). The transfection was performed using Lipofectamine $^{\mathrm{TM}} 2000$ (Invitrogen; Thermo Fisher Scientific, Inc.), according to the manufacturer's protocol.

Reverse transcription-quantitative polymerase chain reaction (RT-qPCR). The total RNA was extracted from tissues and cells with TRIzol reagent (Invitrogen; Thermo Fisher Scientific, Inc.). To detect miR-130a expression, total RNA was reverse transcribed to cDNA using M-MLV (Promega Corporation, Madison, WI, USA). qPCR analysis for miR-130a was performed in triplicate with SYBR ${ }^{\circledR}$ Premix Ex Taq ${ }^{\mathrm{TM}}$ II s (Takara Biotechnology Co., Ltd., Dalian, China) according to the manufacturer's protocol with the ABI 7500 Real-time PCR detection system (Applied Biosystems; Thermo Fisher Scientific, Inc.). The thermocycling conditions were as follows: $5 \mathrm{~min}$ at $95^{\circ} \mathrm{C}$, followed by 40 cycles of $95^{\circ} \mathrm{C}$ for $30 \mathrm{sec}$ and $65^{\circ} \mathrm{C}$ for $45 \mathrm{sec}$. To determine FOXF2 mRNA expression, cDNA was synthesized using the Taqman RT reagents (Applied Biosystems; Thermo Fisher Scientific, Inc.) followed by real-time PCR analysis with $\mathrm{SYBR}^{\circledR}$ Premix Ex Taq ${ }^{\mathrm{TM}}$ II s (Takara Biotechnology Co., Ltd.). The amplification was performed with cycling conditions as follows: $5 \mathrm{~min}$ at $95^{\circ} \mathrm{C}$, followed by 40 cycles of $95^{\circ} \mathrm{C}$ for $30 \mathrm{sec}$ and $65^{\circ} \mathrm{C}$ for $45 \mathrm{sec}$. The primers were designed as follows: miR-130a, 5'-GGC AGTGCAATGTTAAAAG-3' (forward) and 5'-CAGTGCGTG TCGTGGAGT-3' (reverse); U6, 5'-CGCAAGGATGACACG-3' (forward) and 5'-GAGCAGGCTGGAGAA-3' (reverse); FOX F2, 5'-TCGCTGGAGCAGAGCTACTT-3' (forward) and 5'-CCCATTGAAGTTGAGGACGA-3' (reverse); and GAPDH,
5'-CGGAGTCAACGGATTTGGTCGTAT-3' (forward) and 5'-AGCCTTCTCCATGGTGGTGAAGAC-3' (reverse). The fold change in miR-130a or FOXF2 mRNA expression was calculated using the $2^{-\Delta \Delta C q}$ method following normalization to U6 or GAPDH, respectively (20).

MTT assay. The effect of miR-130a on CRC cell proliferation was measured by MTT assay (Sigma-Aldrich; Merck KGaA, Darmstadt, Germany) assay. After transfection for $24 \mathrm{~h}$, transfected cells were collected, counted and seeded at $3 \times 10^{3}$ cells/well in 96-well plates in a final volume of $100 \mu \mathrm{l}$ culture medium/well. For quantitation of cell proliferation, MTT assay was performed after incubation for 1, 2, 3 and 4 days. At the indicated time, $20 \mu 1$ MTT solution $(5 \mathrm{mg} / \mathrm{ml})$ was added to each well. After $4 \mathrm{~h}$ of further incubation, culture medium was removed carefully and $200 \mu$ l dimethyl sulfoxide was added to dissolve the formazan. The optical density (OD) was measured at $490 \mathrm{~nm}$ of each well using a microplate reader (Bio-Rad Laboratories, Inc., Hercules, CA, USA).

Migration and invasion assay. Migration and invasion assays were used to evaluate the effects of miR-130a on migration and invasion capacities of CRC cells. For the migration assay, transfected cells were harvested at $48 \mathrm{~h}$ after transfection, counted and re-suspended in FBS-free culture medium. Subsequently, $5 \times 10^{4}$ cells were seeded in upper chamber of a 24-well Transwell Chamber $(8-\mu \mathrm{m}$ pore size; Corning Incorporated, Corning, NY, USA), and culture medium containing $20 \%$ FBS was placed in the lower chamber as a chemoattractant. After incubation at $37^{\circ} \mathrm{C}$ for $48 \mathrm{~h}$, the non-migrated cells were removed with a cotton tip, and migrated cells were stained with $0.5 \%$ crystal violet at room temperature for $20 \mathrm{~min}$ and counted under a microscope (x200 magnification; IX51; Olympus Corporation, Tokyo, Japan). An invasion assay was performed similar to the migration assay except that Transwell chambers were coated with Matrigel (BD Biosciences, San Jose, CA, USA). All experiments were performed in triplicate.

miRNA target prediction. TargetScan (www.targetscan.org/), PicTar (pictar.mdc-berlin.de/) and miRanda (www.microrna. org/) were used to predict the potential target genes of miR-130a.

Luciferase reporter assay. pGL3-FOXF2-3'UTR wild-type (Wt) and pGL3-FOXF2-3'UTR mutant (Mut) were obtained from Shanghai GenePharma Co., Ltd. (Shanghai, China). For the luciferase reporter assay, the HEK293T cells were transfected with miR-130a inhibitor or NC inhibitor, together with pGL3-FOXF2-3'UTR Wt or pGL3-FOXF2-3'UTR Mut using Lipofectamine 2000. At $48 \mathrm{~h}$ post-transfection, the cells were harvested, and then luciferase activities were measured by using Dual-Luciferase Reporter Assay system (Promega Corporation), according to the manufacturer's protocol. The relative luciferase activities were determined by normalizing to Renilla luciferase activities.

Western blotting. Cells were harvested, and total protein was exacted from transfected cells following lysing in radioimmunopreciptation buffer (Thermo Fisher Scientific, Inc.) at 
Table I. Correlation between clinicopathological features and miR-130a expression in colorectal cancer.

\begin{tabular}{lrrrr}
\hline & & \multicolumn{2}{c}{$\begin{array}{c}\text { miR-130a } \\
\text { expression }\end{array}$} & \\
\cline { 3 - 4 } Variable & Total & High & Low & P-value \\
\hline Sex & & & & 0.974 \\
$\quad$ Male & 33 & 18 & 15 & \\
Female & 20 & 11 & 9 & \\
Age & & & & 0.859 \\
$\quad<60$ years & 25 & 14 & 11 & \\
$\geq 60$ years & 28 & 15 & 13 & \\
TNM stage & & & & 0.002 \\
I-II & 32 & 12 & 20 & \\
III-IV & 21 & 17 & 4 & \\
Tumor size & & & & 0.134 \\
$\quad<5$ cm & 34 & 16 & 18 & \\
$\geq 5$ cm & 19 & 13 & 6 & \\
Lymph node metastasis & & & & 0.001 \\
$\quad$ No & 34 & 13 & 21 & \\
$\quad$ Yes & 19 & 16 & 3 & \\
\hline
\end{tabular}

miR, microRNA.

$72 \mathrm{~h}$ after transfection. The concentration of total protein was detected using a Bicinchoninic Acid assay kit (Thermo Fisher Scientific, Inc.) based on the manufacturer's protocol. Equal amounts of protein $(30 \mu \mathrm{g})$ were separated by $10 \%$ sodium dodecyl sulfate-polyacrylamide gel electrophoresis, transferred to polyvinylidene difluoride membrane (Bio-Rad Laboratories, Inc.) and blocked with 5\% non-fat dry milk in TBS containing $0.1 \%$ Tween-20 (TBST). Subsequently, the membranes were incubated at $4^{\circ} \mathrm{C}$ overnight with primary antibodies, including mouse anti-human monoclonal FOXF2 (sc-101043; 1:1,000; Santa Cruz Biotechnology, Inc., Dallas, TX, USA) and mouse anti-human monoclonal GAPDH (sc-47724; 1:1,000; Santa Cruz Biotechnology, Inc.). Subsequently, the membranes were washed with TBST three times and probed with a horseradish peroxidase-conjugated goat anti-mouse secondary antibody (sc-2005; 1:1,000; Santa Cruz Biotechnology, Inc.) for $2 \mathrm{~h}$ at room temperature. The proteins were visualized with enhanced chemiluminescence-plus reagents (GE Healthcare, Chicago, IL, USA), following the manufacturer's protocol. The density of the protein bands was determined using ImageJ software (version 1.49; National Institutes of Health, Bethesda, $\mathrm{MD}, \mathrm{USA}$ ), and values were normalized to GAPDH.

Statistical analysis. All the data are expressed as the mean \pm standard deviation. Data were compared with Student's t-test or one-way analysis of variance followed by Student-Newman-Keuls post hoc test, using SPSS software version 17 (SPSS, Inc., Chicago, IL, USA). The relationship between miR-130a expression level and clinicopathological features of CRC was analyzed using Pearson's $\chi^{2}$ test. $\mathrm{P}<0.05$ was considered to indicate a statistically significant difference.
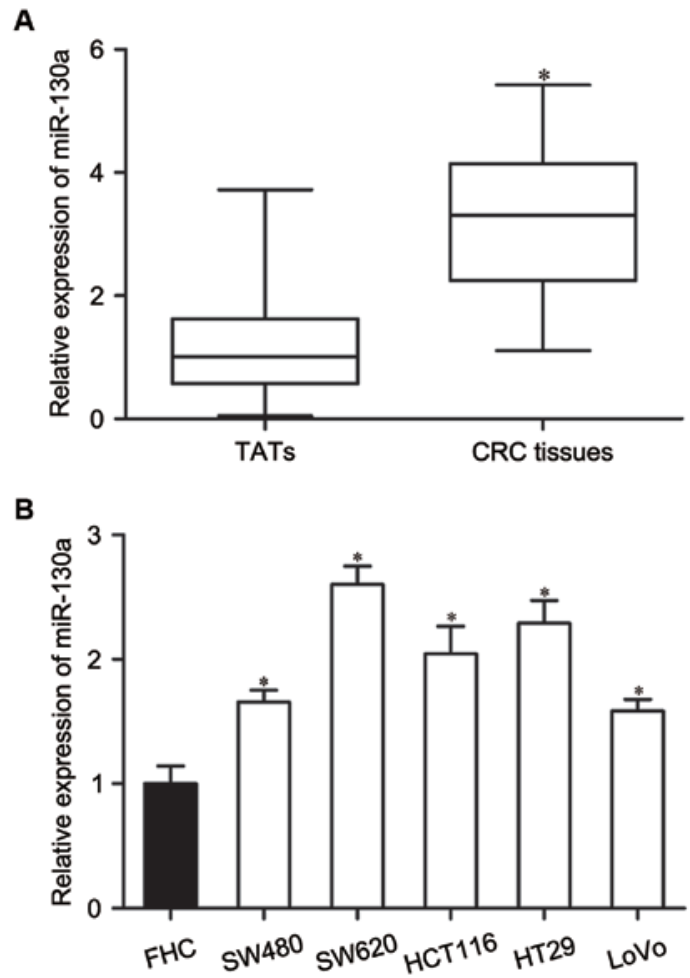

Figure 1. miR-130a is overexpressed in CRC. (A) miR-130a expression levels in CRC tissues and matched TATs. (B) The expression of miR-130a in five CRC cell lines (SW480, SW620, HCT116, HT29 and LoVo) and the FHC human normal colon epithelium cell line (FHC). Data are expressed as the mean \pm standard deviation. ${ }^{*} \mathrm{P}<0.05$ vs. respective control. TATs, tumor adjacent tissues; CRC, colorectal cancer; miR, microRNA.

\section{Results}

miR-130a is upregulated in CRC tissues and cell lines. miR-130a expression in CRC tissues and matched TATs was measured using RT-qPCR. As presented in Fig. 1A, miR-130a expression was significantly upregulated in CRC tissues compared with in matched TATs $(\mathrm{P}<0.05)$. To further assess the clinical significance of miR-130a in CRC, the correlation between clinicopathological features and miR-130a expression in CRC was analyzed. The results demonstrated that miR-130a expression was obviously correlated with TNM stage $(\mathrm{P}=0.002)$ and lymph node metastasis $(\mathrm{P}=0.001)$, while there was no correction with age, sex and tumor size (Table I).

miR-130a expression levels were also detected in CRC cell lines (SW480, SW620, HCT116, HT29 and LoVo) and the FHC human normal colon epithelium cell line. miR-130a expression levels were relatively higher in all examined CRC cell lines than in the FHC cell line (Fig. $1 \mathrm{~B}, \mathrm{P}<0.05$ ). These results suggested that miR-130a may serve as an oncogene in CRC.

Inhibition of miR-130a expression inhibits CRC cell proliferation, migration and invasion. To explore the roles of miR-130a in the CRC, miR-130a inhibitor or NC inhibitor was transfected into SW620 and HT29 cells. The expression of miR-130a was downregulated following transfection with the miR-130a inhibitor compared with the NC inhibitor in both cell lines, as detected by RT-qPCR (Fig. 2A, P<0.05). The effects of miR-130a on growth and metastasis of CRC cells were assessed by MTT, migration and invasion assays, 

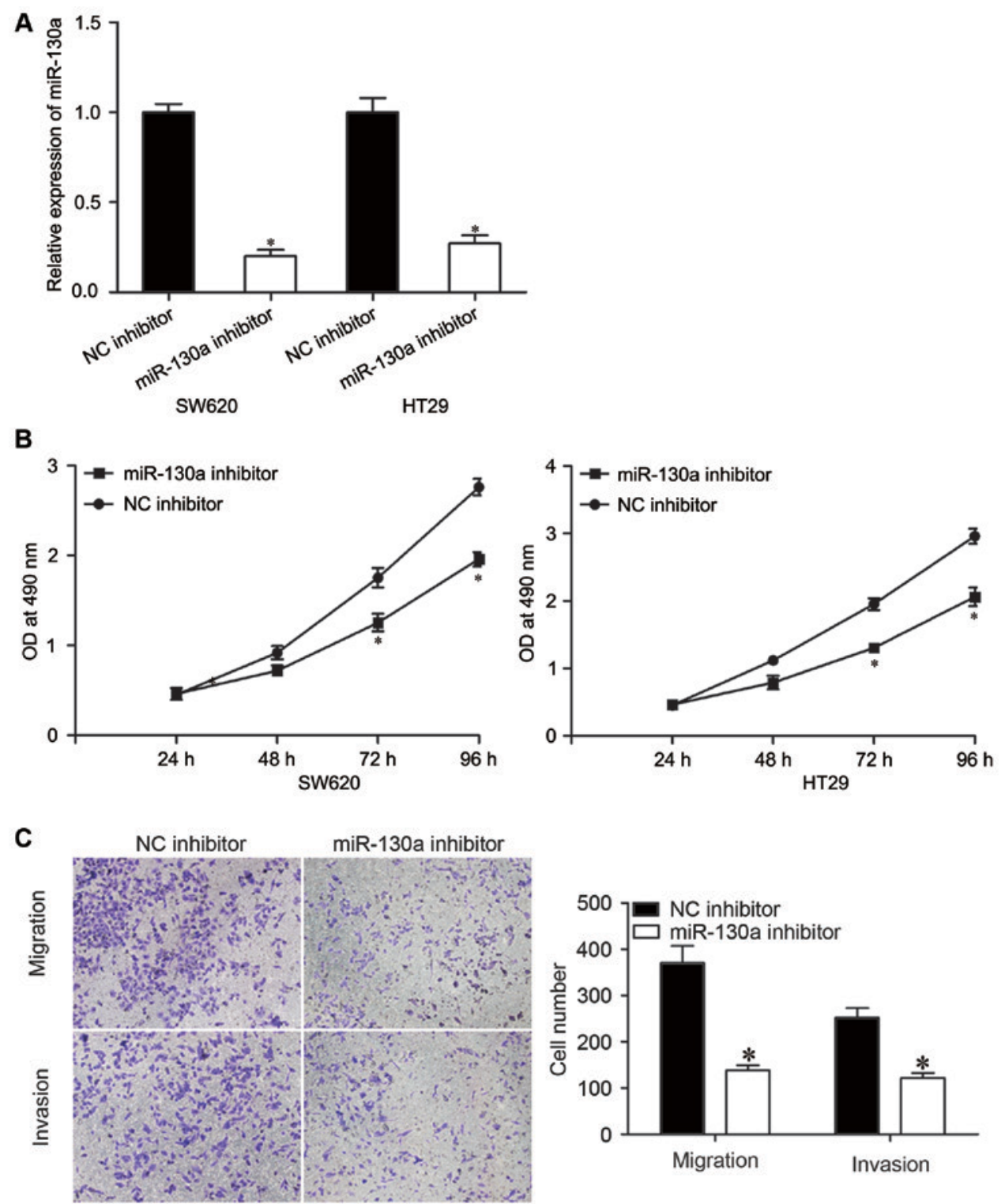

SW620
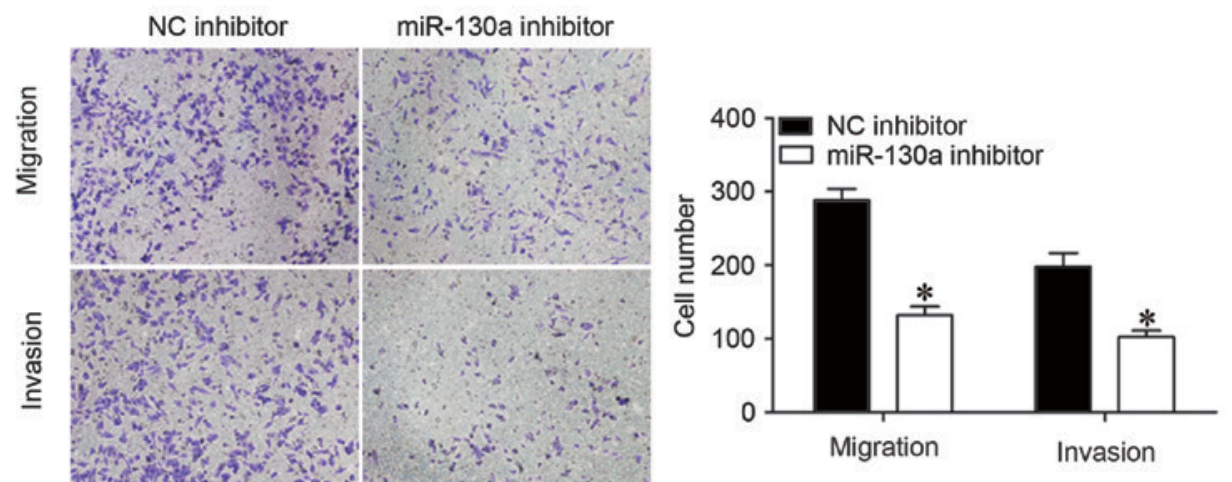

HT29

Figure 2. Inhibition of miR-130a inhibits CRC cell proliferation, migration and invasion. (A) Reverse transcription-quantitative polymerase chain reaction was performed to determine miR-130a expression levels in SW620 and HT29 cells after transfection with miR-130a or NC inhibitors. (B) MTT assay was used to investigate the effect of the miR-130a inhibitor on SW620 and HT29 cell proliferation. (C) The migration and invasion capacity of SW620 and HT29 cells transfected with miR-130a inhibitor or $\mathrm{NC}$ inhibitor was evaluated by migration and invasion assay. Data are expressed as the mean \pm standard deviation. ${ }^{*} \mathrm{P}<0.05$ vs. NC inhibitor. CRC, colorectal cancer; miR, microRNA; NC, negative control; OD, optical density.

respectively. The results of the MTT assay revealed that inhibition of miR-130a decreased SW620 and HT29 cell proliferation (Fig. 2B, $\mathrm{P}<0.05$ ). The migration and invasion assay revealed that downregulation of miR-130a reduced the migratory and invasive abilities of SW620 and HT29 cells compared with the NC inhibitor cells (Fig. $2 \mathrm{C}, \mathrm{P}<0.05$ ). These 
A

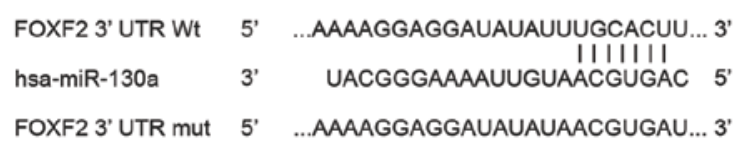

C

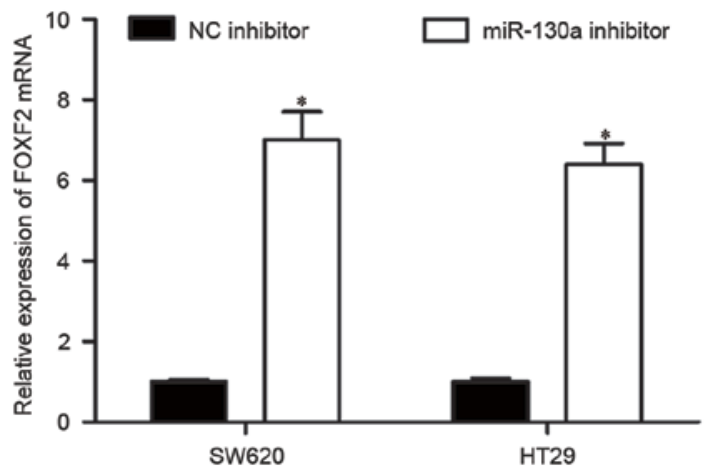

B

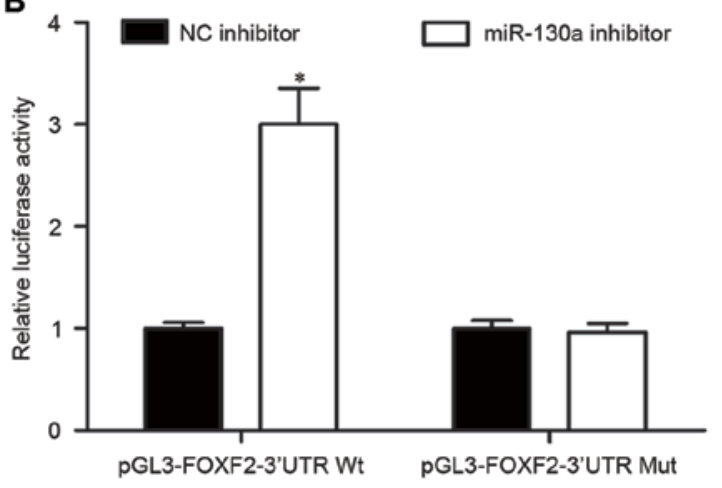

D
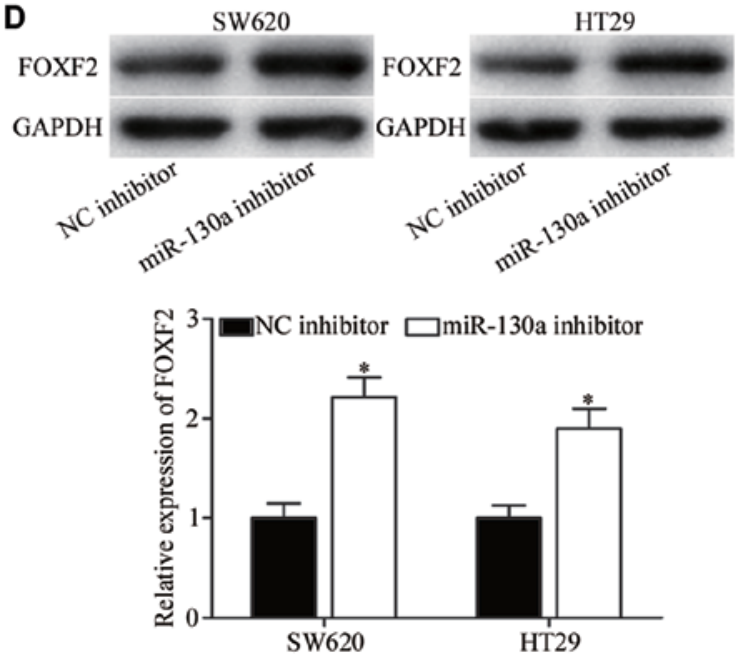

Figure 3. FOXF2 is the direct downstream target gene of miR-130a in colorectal cancer. (A) The putative miR-130a binding sites in the 3'UTR of FOXF2 are presented. A mutation was generated on the FOXF2 3'UTR sequence in the complementary site for the seed region of miR-130a. (B) Luciferase reporter assay of HEK293T cells transfected with pGL3-FOXF2-3'UTR Wt or pGL3-FOXF2-3'UTR Mut reporter plasmid, along with miR-130a inhibitor or NC inhibitor. (C) Reverse transcription-quantitative polymerase chain reaction analysis of FOXF2 mRNA expression levels in SW620 and HT29 cells transfected with miR-130a inhibitor or NC inhibitor. (D) Western blot analysis of FOXF2 protein expression levels in SW620 and HT29 cells transfected with miR-130a inhibitor or NC inhibitor. Data are expressed as the mean \pm standard deviation. "P<0.05 vs. NC inhibitor. miR, microRNA; NC, negative control; OD, optical density; FOXF2, forkhead box F2; Wt, wild-type; Mut, mutant.

findings suggested that inhibition of miR-130a repressed the proliferation, migration and invasion of CRC cells.

FOXF2 is the direct downstream target gene of miR-130a in $C R C$. To elucidate the underlying molecular mechanisms by which miR-130a exerts its carcinogenic functions, the target genes of miR-130a were predicted using TargetScan, PicTar and miRanda. FOXF2 was selected as a potential target of miR-130a based on putative target sequences at position 663-669 of the FOXF2-3'UTR (Fig. 3A). To determine whether FOXF2 was a direct target of miR-130a, a luciferase reporter assay was performed. As presented in Fig. 3B, the luciferase activities of the pGL3-FOXF2-3'UTR Wt reporter plasmid was significantly upregulated following miR-130a inhibitor transfection $(\mathrm{P}<0.05)$. However, pGL3-FOXF2-3'UTR Mut reporter plasmid completely abolished the upregulation, indicating that miR-130a directly targets FOXF2.

Furthermore, RT-qPCR and western blotting were performed to determine if miR-130a could regulate FOXF2 expression in CRC cells. The results revealed that FOXF2 mRNA (Fig. 3C, $\mathrm{P}<0.05$ ) and protein (Fig. 3D, $\mathrm{P}<0.05$ ) expression levels were significantly upregulated by miR-130a inhibition in SW620 and HT29 cells. These results suggested that FOXF2 was the direct downstream target gene of miR-130a in CRC.

Downregulation of FOXF2 partially reversed the effects of miR-130a in CRC cells. To further explore the functional consequence of the miR-130a/FOXF2 interactions in CRC, FOXF2 siRNA or NC siRNA was transfected into SW620 and HT29 cells. The mRNA (Fig. 4A, P<0.05) and protein (Fig. 4B, $\mathrm{P}<0.05)$ expression levels of FOXF2 in SW620 and HT29 cells were downregulated, as determined by RT-qPCR and western blotting, respectively. Additionally, the proliferation, migration and invasion of CRC cells was assessed by MTT, migration and invasion assays. Co-transfection of FOXF2 siRNA partially reversed the effects caused by miR-130a inhibitor in CRC cell proliferation (Fig. $4 \mathrm{C}, \mathrm{P}<0.05$ ), and migration and invasion (Fig. 4D, $\mathrm{P}<0.05$ ). Collectively, these data indicated that downregulation of miR-130a inhibits proliferation, migration and invasion of CRC cells through upregulation of FOXF2.

\section{Discussion}

Tumorigenesis of many cells is relevant to the dysregulation of miRNAs, indicating that the abnormal expression of 
A

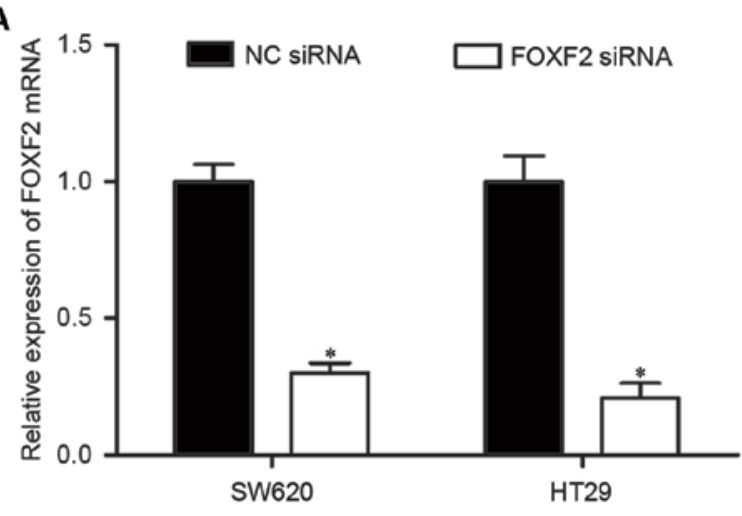

C

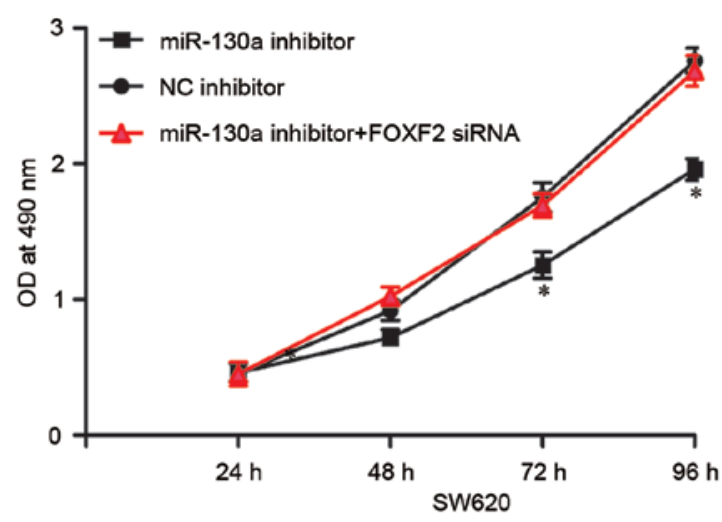

D
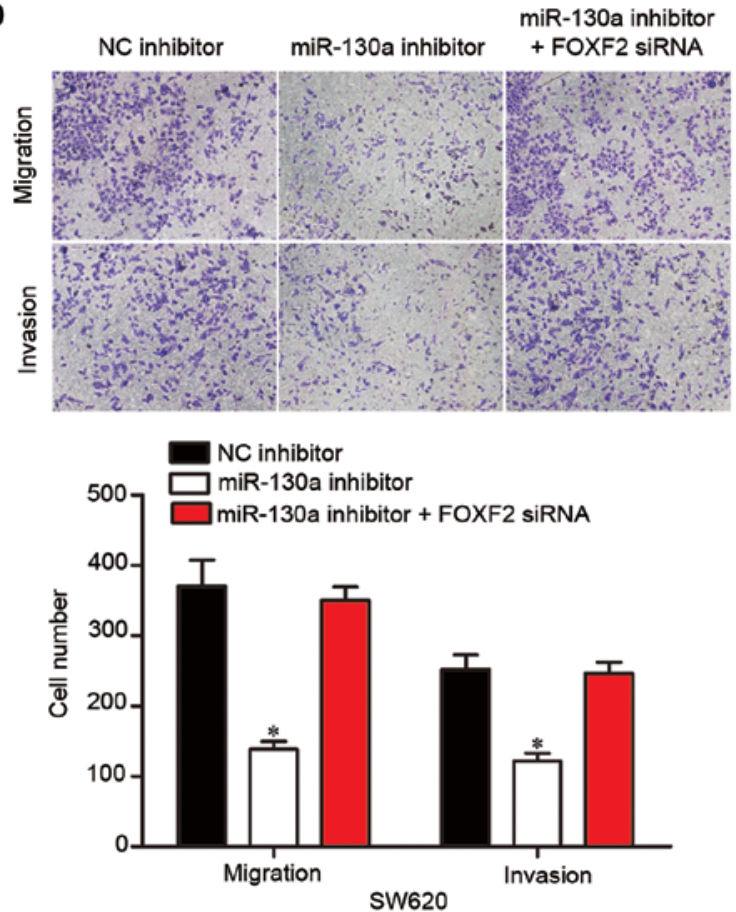

B
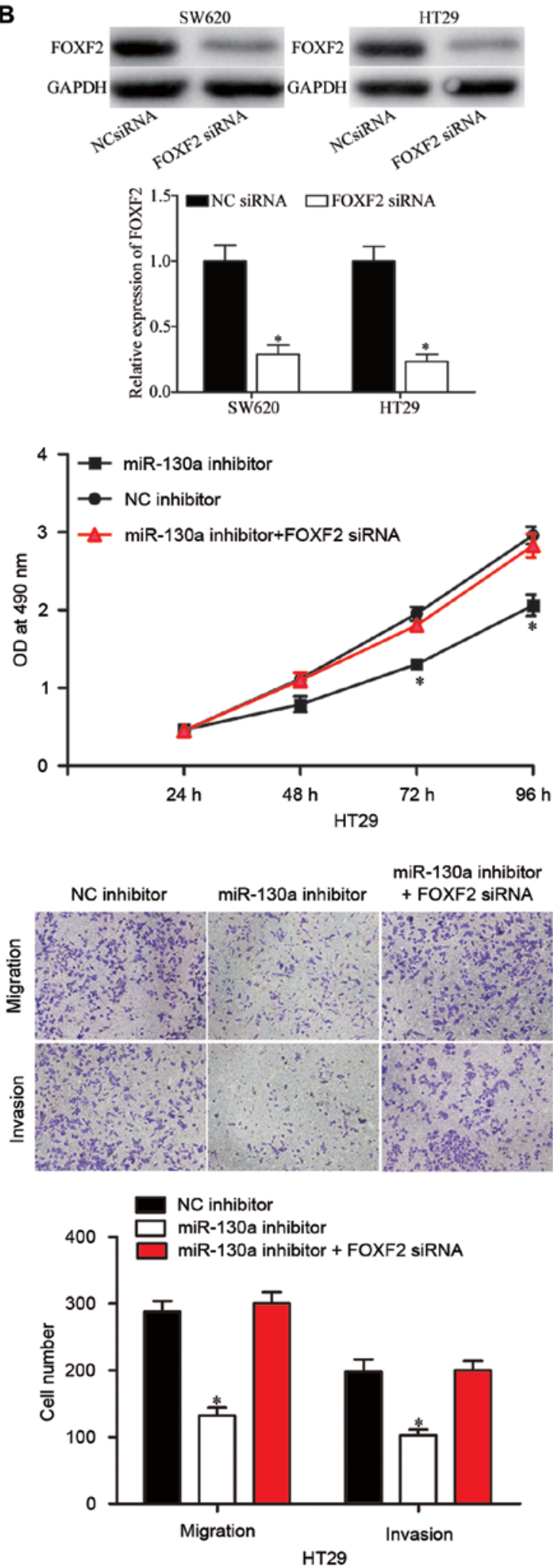

Figure 4. Downregulation of FOXF2 reverses the effects of miR-130a inhibitor in colorectal cancer cells. (A) Reverse transcription-quantitative polymerase chain reaction analysis of FOXF2 mRNA expression levels in SW620 and HT29 cells transfected with FOXF2 siRNA or NC siRNA. (B) Western blot analysis of FOXF2 protein expression levels in SW620 and HT29 cells transfected with FOXF2 siRNA or NC siRNA. Downregulation of FOXF2 partially reversed the effects of miR-130a inhibitor in CRC cell (C) proliferation, and (D) migration and invasion. Data are expressed as the mean \pm standard deviation. ${ }^{*} \mathrm{P}<0.05$ vs. NC. miR, microRNA; NC, negative control; OD, optical density; FOXF2, forkhead box F2; siRNA, small interfering RNA.

miRNAs may serve important functions in carcinogenesis and tumor development (21). To the best of our knowledge, the present study was the first to demonstrate that miR-130a serves as a carcinogenic miRNA by directly targeting FOXF2 in CRC. Firstly, miR-130a was significantly upregulated in CRC tissues and cell lines. Secondly, the expression levels of miR-130a were markedly correlated with TNM stage and lymph node metastasis of CRC patients. Thirdly, inhibition 
of miR-130a inhibited CRC cells proliferation, migration and invasion. Additionally, FOXF2 was validated as a direct target of miR-130a in bioinformatics analysis, luciferase reporter assays, RT-qPCR and western blotting. Finally, inhibition of FOXF2 partially reversed the effects induced by miR-130a under-expression in CRC cells. Therefore, miR-130a may be highlighted as a novel target for the therapeutic treatment of patients with CRC.

Previous studies reported the abnormal expression of miR-130a in human cancers; the expression levels of miR-130a were reduced in breast cancer (22), hepatocellular carcinoma (23), non-small cell lung cancer (24), prostate carcinoma (25) and chronic myeloid leukemia (26). However, elevated miR-130a expression has been identified in gastric cancer (27), and cisplatin resistance of ovarian cancer (28). Its expression levels were demonstrated to be closely associated with clinicopathological features of patients with malignant tumors. For example, in gastric cancer, patients with high miR-130a expression levels had significantly poorer overall survival compared with the low-miR-130a group (27). In hepatocellular carcinoma, miR-130a expression is associated with sex, HBsAg status, tumor size and tumor-node-metastasis stage. Kaplan-Meier analysis revealed that hepatocellular carcinoma cases with low miR-130a expression had a poorer overall survival than patients with high miR-130a expression (23). Furthermore, multivariate Cox regression analysis demonstrated that miR-130a expression was an independent prognostic factor for overall survival (23). In cervical cancer, survival analysis revealed that higher expression of miR-130a was significantly correlated with poor disease-free survival (29). In non-small cell lung cancer, miR-130a is correlated with poor prognosis and increased tumor stage and metastasis (30). These studies suggested that miR-130a might be a useful marker for the diagnosis and prognosis of cancers.

miR-130a has been identified as a tumor suppressor in cancers. Liu et al (31) reported that overexpression of miR-130a inhibits cell detachment, attachment, migration and invasion in gemcitabine-resistant hepatocellular carcinoma cells. Pan et al (22) demonstrated that ectopic miR-130a expression suppresses breast cancer cell growth, migration and invasion. Lin et al (30) demonstrated that in non-small cell lung cancer, restoration of miR-130a expression in macrophages resulted in a significantly increased production of proinflammatory cytokines, whereas deletion of miR-130a impaired M2-associated gene expression and led to an M1-biased response (30). In prostate cancer, miR-130a overexpression decreases prostate cancer cell proliferation and increases the sensitivity to paclitaxel (32). However, miR-130a has been demonstrated to serve carcinogenic functions in gastric cancer. Enforced miR-130a expression promotes gastric cancer carcinogenesis by enhancing cell proliferation migration and invasion (27). These conflicting studies demonstrated that miR-130a serves as an oncogene in certain cancers, and a tumor suppressor in others. This contradiction may be explained by the 'imperfect complementarity' of the interactions between miRNAs and target genes (33).

miRNAs may function as tumor suppressors or oncogenes, mainly depending on their target genes. The present study investigated the molecular mechanism by which downregulation of miR-130a inhibits CRC cell growth and metastasis. TargetScan, PicTar and miRanda software were used to predict the potential target genes of miR-130a. The bioinformatics analysis revealed that FOXF2 was a potential target of miR-130a based on putative target sequences at position 663-669 of the FOXF2-3'UTR. A luciferase reporter assay verified that miR-130a directly targets the 3'UTR of FOXF2. RT-qPCR and western blotting indicated that miR-130a regulates endogenous FOXF2 expression at both the mRNA and protein levels in CRC. Notably, knockdown of FOXF2 partially reversed the effects of miR-130a-underexpression in CRC cells. These results suggested that miR-130a exerts its carcinogenic functions in CRC carcinogenesis and progression, at least in part, by regulation of FOXF2 expression.

In conclusion, the present study revealed that miR-130a is an oncogene in CRC. miR-130a expression levels were frequently increased in CRC tissues and cell lines, and expression levels of miR-130a were significantly associated with TNM stage and lymph node metastasis of CRC patients. In functional studies, downregulation of miR-130a inhibited CRC cells proliferation, migration and invasion. Notably, FOXF2 was validated as a direct and functional target of miR-130a in CRC. These findings suggested that miR-130a could be a potential prognostic marker and therapeutic target for patients with CRC.

\section{References}

1. Center MM, Jemal A, Smith RA and Ward E: Worldwide variations in colorectal cancer. CA Cancer J Clin 59: 366-378, 2009.

2. Markowitz SD and Bertagnolli MM: Molecular origins of cancer: Molecular basis of colorectal cancer. N Engl J Med 361: 2449-2460, 2009.

3. Chi Y and Zhou D: MicroRNAs in colorectal carcinoma-from pathogenesis to therapy. J Exp Clin Cancer Res 35: 43, 2016.

4. Yu H, Gao G, Jiang L, Guo L, Lin M, Jiao X, Jia W and Huang J: Decreased expression of miR-218 is associated with poor prognosis in patients with colorectal cancer. Int J Clin Exp Pathol 6: 2904-2911, 2013

5. Haggar FA and Boushey RP: Colorectal cancer epidemiology: Incidence, mortality, survival and risk factors. Clin Colon Rectal Surg 22: 191-197, 2009.

6. Van Cutsem E, Cervantes A, Nordlinger B and Arnold D; ESMO Guidelines Working Group: Metastatic colorectal cancer: ESMO clinical practice guidelines for diagnosis, treatment and follow-up. Ann Oncol 25 (Suppl 3): iii1-9, 2014.

7. Bartel DP: MicroRNAs: Target recognition and regulatory functions. Cell 136: 215-233, 2009.

8. Kasinski AL and Slack FJ: Epigenetics and genetics. MicroRNAs en route to the clinic: Progress in validating and targeting microRNAs for cancer therapy. Nat Rev Cancer 11: 849-864, 2011.

9. He L and Hannon GJ: MicroRNAs: Small RNAs with a big role in gene regulation. Nat Rev Genet 5: 522-531, 2004.

10. Valencia-Sanchez MA, Liu J, Hannon GJ and Parker R: Control of translation and mRNA degradation by miRNAs and siRNAs. Genes Dev 20: 515-524, 2006.

11. Winter J, Jung S, Keller S, Gregory RI and Diederichs S: Many roads to maturity: microRNA biogenesis pathways and their regulation. Nat Cell Biol 11: 228-234, 2009.

12. Forman JJ, Legesse-Miller A and Coller HA: A search for conserved sequences in coding regions reveals that the let-7 microRNA targets Dicer within its coding sequence. Proc Natl Acad Sci USA 105: 14879-14884, 2008.

13. Calin GA and Croce CM: MicroRNA signatures in human cancers. Nat Rev Cancer 6: 857-866, 2006.

14. Esquela-Kerscher A and Slack FJ: Oncomirs-microRNAs with a role in cancer. Nat Rev Cancer 6: 259-269, 2006.

15. Manikandan J, Aarthi JJ, Kumar SD and Pushparaj PN: Oncomirs: The potential role of non-coding microRNAs in understanding cancer. Bioinformation 2: 330-334, 2008.

16. Zhou H, Guo W, Zhao Y, Wang Y, Zha R, Ding J, Liang L, Hu J, Shen H, Chen Z, et al: MicroRNA-26a acts as a tumor suppressor inhibiting gallbladder cancer cell proliferation by directly targeting HMGA2. Int J Oncol 44: 2050-2058, 2014. 
17. Huang J, Zhang SY, Gao YM, Liu YF, Liu YB, Zhao ZG and Yang K: MicroRNAs as oncogenes or tumour suppressors in oesophageal cancer: Potential biomarkers and therapeutic targets. Cell Prolif 47: 277-286, 2014.

18. Long HC, Gao X, Lei CJ, Zhu B, Li L, Zeng C, Huang JB and Feng JR: miR-542-3p inhibits the growth and invasion of colorectal cancer cells through targeted regulation of cortactin. Int J Mol Med 37: 1112-1218, 2016.

19. Qiu Y, Yu H, Shi X, Xu K, Tang Q, Liang B, Hu S, Bao Y, Xu J, Cai J, et al: microRNA-497 inhibits invasion and metastasis of colorectal cancer cells by targeting vascular endothelial growth factor-A. Cell Prolif 49: 69-78, 2016.

20. Livak KJ and Schmittgen TD: Analysis of relative gene expression data using real-time quantitative PCR and the 2(-Delta Delta C(T)) method. Methods 25: 402-408, 2001.

21. Xiao R, Li C and Chai B: miRNA-144 suppresses proliferation and migration of colorectal cancer cells through GSPT1. Biomed Pharmacother 74: 138-144, 2015.

22. Pan Y, Wang R, Zhang F, Chen Y, Lv Q, Long G and Yang K: MicroRNA-130a inhibits cell proliferation, invasion and migration in human breast cancer by targeting the RAB5A. Int J Clin Exp Pathol 8: 384-393, 2015.

23. Li B, Huang P, Qiu J, Liao Y,Hong J and Yuan Y: MicroRNA-130a is down-regulated in hepatocellular carcinoma and associates with poor prognosis. Med Oncol 31: 230, 2014

24. Acunzo M, Visone R, Romano G, Veronese A,Lovat F, Palmieri D, Bottoni A, Garofalo M, Gasparini P, Condorelli G, et al: miR-130a targets MET and induces TRAIL-sensitivity in NSCLC by downregulating miR-221 and 222. Oncogene 31: 634-642, 2012.

25. Boll K, Reiche K, Kasack K, Mörbt N, Kretzschmar AK, Tomm JM, Verhaegh G, Schalken J, von Bergen M, Horn F and Hackermüller J: MiR-130a, miR-203 and miR-205 jointly repress key oncogenic pathways and are downregulated in prostate carcinoma. Oncogene 32: 277-285, 2013.
26. Zhu X, Zhao H, Lin Z and Zhang G: Functional studies of miR-130a on the inhibitory pathways of apoptosis in patients with chronic myeloid leukemia. Cancer Gene Ther 22: 573-580, 2015.

27. Jiang H, Yu WW, Wang LL and Peng Y: miR-130a acts as a potential diagnostic biomarker and promotes gastric cancer migration, invasion and proliferation by targeting RUNX3. Oncol Rep 34: 1153-1161, 2015

28. Yang LY, Wang HJ, Jia XB, Wang X, Luo J and Zhang XY: Expression of miR-130a in cisplatin resistant cell lines of ovarian cancer. Sichuan Da Xue Xue Bao Yi Xue Ban 43: 60-64, 2012 (In Chinese).

29. He L, Wang HY, Zhang L, Huang L, Li JD, Xiong Y, Zhang MY, Jia WH, Yun JP, Luo RZ and Zheng M: Prognostic significance of low DICER expression regulated by miR-130a in cervical cancer. Cell Death Dis 5: e1205, 2014.

30. Lin L, Lin H, Wang L, Wang B, Hao X and Shi Y: miR-130a regulates macrophage polarization and is associated with non-small cell lung cancer. Oncol Rep 34: 3088-3096, 2015.

31. Liu Y, Li Y, Wang R, Qin S, Liu J, Su F, Yang Y, Zhao F, Wang Z and Wu Q: MiR-130a-3p regulates cell migration and invasion via inhibition of Smad4 in gemcitabine resistant hepatoma cells. J Exp Clin Cancer Res 35: 19, 2016.

32. Fujita Y, Kojima T, Kawakami K, Mizutani K, Kato T, Deguchi T and Ito M: miR-130a activates apoptotic signaling through activation of caspase- 8 in taxane-resistant prostate cancer cells. Prostate 75: 1568-1578, 2015.

33. Yu Z, Ni L, Chen D, Zhang Q, Su Z, Wang Y, Yu W, Wu X, Ye J, Yang S, et al: Identification of miR-7 as an oncogene in renal cell carcinoma. J Mol Histol 44: 669-677, 2013. 